\title{
Impact of Shared Decision-making on Compliance with Oral Nutritional Supplements and Quality of Life Among Elderly Patients with Cirrhosis
}

\author{
Li Guanhong ${ }^{1,2}$, Liang Dongxiu ${ }^{1,2}$, Chen Zhaolin ${ }^{1,2, *}$ \\ ${ }^{1}$ Department of Gastroenterology, the First Affiliated Hospital of Jinan University, Guangzhou, China \\ ${ }^{2}$ School of Nursing, Jinan University, Guangzhou, China
}

Email address:

191426127@qq.com (Li Guanhong),286630107@qq.com (Liang Dongxiu),2009gzjnu@163.com (Chen Zhaolin)

${ }^{*}$ Corresponding author

\section{To cite this article:}

Li Guanhong, Liang Dongxiu, Chen Zhaolin. Impact of Shared Decision-making on Compliance with Oral Nutritional Supplements and Quality of Life Among Elderly Patients with Cirrhosis. American Journal of Nursing Science. Vol. 9, No. 3, 2020, pp. 97-101. doi: 10.11648/j.ajns.20200903.12

Received: March 12, 2020; Accepted: March 26, 2020; Published: April 7, 2020

\begin{abstract}
Objective: To investigate the effect of shared decision-making on compliance with oral nutrition supplementation (ONS) and quality of life of among elderly patients ( $\geq 60$ years) with cirrhosis. Methods: This study used an action research design that tested the intervention of shared decision-making among elderly patients with cirrhosis. It measured how this intervention influenced compliance with oral nutrition supplements and quality of life. Sixty-eight elderly patients with cirrhosis who were hospitalized in our department from May 2019 to November 2019 were randomly divided into two groups: the control group the observation group. The total number of participants in each group was 34 . The control group received routine care. The observation group received shared decision-making nutrition care in addition to routine care. Results: After the intervention, patients in the observation group had higher oral nutritional compliance and quality of life scores than the control group and had a statistical significance $(\mathrm{P}<0.05)$. Conclusion: After the implementation of Shared decision nutrition care for 34 elderly patients with cirrhosis, the patients' ONS compliance and quality of life were greatly improved. At present, there are relatively few researches on the application of Shared decision in digestive system diseases, Hope to play a certain role of reference or guidance.
\end{abstract}

Keywords: Shared Decision-making, Cirrhosis, Elderly Patients, Oral Nutritional Supplementation, Compliance, Quality of Life

\section{Introduction}

In recent years, living standards have steadily improved. In China, the proportion of the population aged 60 years and over (60 years or more population), is also increasing. Simultaneously, the number of elderly patients with cirrhosis has increased every year. Cirrhosis is a late stage of irreversible scarring on the liver. It is a tenacious disease that affects the digestive system and can persist for years [1]. Meanwhile, the majority of China's elderly population lives by themselves and has irregular dietary habits. Furthermore, digestive system diseases can easily affect the appetite of the elderly, leading to malnutrition. It has been shown that [2] malnutrition can severely affect quality of life, and even the prognosis of elderly patients. The majority of clinical nutrition experts use oral nutritional supplements (ONS) to treat malnutrition in elderly patients with cirrhosis [3]. However, as patients and their families lack awareness of nutrition's importance, ONS compliance and patient quality of life are severely affected [4]. Therefore, the search for interventions that improve ONS compliance and quality of life among elderly patients with cirrhosis is urgently needed. Shared decision-making between physicians and patients was first proposed in the 1990s [5]. It refers to the process by which healthcare workers and the patient come to an agreement based on the patient's preferences and values in the absence of a best treatment option [6]. With increasing medical awareness among patients and their families, studies have 
shown that patient compliance is improved when the patients and their families engage in healthcare plans. Few studies have reported on the application of shared decision-making for digestive system diseases. Therefore, this study conducted the relevant research and the findings are presented herein.

\section{Materials and Methods}

\subsection{Baseline Characteristics}

A total of 68 elderly patients with cirrhosis who were admitted to our hospital department from May 2019 to November 2019 were included in this study. Inclusion criteria: (1) Patients aged $\geq 60$ years who met the diagnostic criteria for cirrhosis [7]; (2) Patients with a clear consciousness and normal mental state, and who had a Nutrition Risk Screening 2002 (NRS-2002) score $\geq 3$; (3) Patients who understood the purpose of this study, provided informed consent, and signed the written informed consent form. Exclusion criteria: (1) Patients with concomitant severe diseases; (2) Concurrent participation in other trials; (3) Unwilling to participate or not cooperative. A total of 68 patients met the above inclusion and exclusion criteria and were randomly divided into the control group and the observation group of 34 patients each using a random number table. The observation group contained 20 males and 14 females aged between 60 and 79 years, with a mean age of $69.23 \pm 3.2$ years. The duration of disease ranged from 3 to 19 years, with a mean of $9.2 \pm 3.7$ years. The number of elderly patients with regular and irregular dietary habits were 16 and 18 , respectively. The control group contained 18 males and 16 females between 61 and 80 years old, with a mean age of $68.77 \pm 3.4$ years. The duration of disease ranged from 2 to 20 years, with a mean of $9.7 \pm 3.9$ years. The number of elderly patients with regular and irregular dietary habits were both 17 . The baseline characteristics of the two patient groups were not significantly different $(\mathrm{P}>0.05)$.

\subsection{Care Approaches}

\subsubsection{Control Group}

The attending physician and nurse observed the patients' dietary habits. The attending physician prescribed ONS based on the patients' clinical examination results (including parameters from complete blood count and biochemical profile), care records, and recent changes in the patients' conditions. The nurse provided routine care and healthrelated education such as diet and medications, based on the medication orders. Also, the nurses provided patients with detailed explanation of the importance of ONS as a nutritional supplement for cirrhosis.

\subsubsection{Observation Group}

A shared decision-making intervention group was established. The group members were comprised of the attending physician, the head nurse, the attending nurse, the patient, and one family member. The attending nurse acted as the team leader and was responsible for the communication and interactions among group members, as well as monitoring measurement compliance. The head nurse was responsible for supervision and inspection to ensure the accurate implementation of the present study plan. The attending physician and nurse, patient, and family member were responsible for formulating an appropriate implementation plan through conversations. (2) Patients enrolled in the observation group would receive the shared decision-making intervention measures in addition to receiving routine care. The specific plan was implemented by the shared decision-making intervention group. Specifically: 1) After the patients were enrolled, the attending nurse introduced them to other group members and explained the purpose, methodology, and goals of the research. The attending nurse also addressed questions raised by patients and their families in detail and with patience. The patients and their families were provided a relaxed and comfortable environment to facilitate subsequent research work. 2) Group members discussed the reasons for poor compliance with ONS and factors, in their opinion, that impact patients' quality of life. Through analysis, a treatment plan that was acceptable to all members was formulated. The content of the specific plan was included in the data entry and management file at the time of patient enrollment, assessment of patients' nutrition status, formulation of nutrition plans, tracking and supervision of ONS compliance, and recording and comparing patients' quality of life. 3) After discussion, the observation group started to implement the intervention measures according to the agreed plan. The procedures included nutrition screening, assessment, intervention, supervision, evaluation, and follow-up. Meanwhile, an ONS administration manual was developed in reference to clinical guidelines to facilitate the consultation and education among group members at all times. 4) All participants in this study received standardized training to ensure that everyone met the standards with regard to oral communication, ONS administration technique, and health education. 5) The attending nurse provided training in the form of seminars on specific topics, one-on-one lectures, and regular ward rounds based on patients' specific conditions. The concurrent enhancement of ONS awareness by healthcare workers and strengthening supervision of patients' ONS compliance ensured the research could be conducted smoothly.

\subsection{Evaluation Methods}

The quality of life for the two groups was scored by the researchers prior to intervention. After one month of intervention, the ONS compliance and quality of life scores for both groups were assessed, respectively. The instruments used for assessment were as follows: We used a Patient ONS Compliance Questionnaire based on the nutrition guidelines for cancer patients [8]. and in consultation with professors in nutrition-related fields and nutrition nurses, the degree of ONS compliance was graded on a scale of poor to good: poor= 0-6 scoops/day; normal= 7-11 scoops/day; and good= more than 12 scoops/day. To measure quality of life, we used the Quality of Life Scale developed by Luo Jiahong et al [9]. The test-retest reliability was $>0.90$ and the Cronbach's $\alpha$ coefficient was $>0.70$. 


\subsection{Statistical Analysis}

All data were analyzed using the SPSS 22.0 statistical software. Quantitative data were expressed as $\mathrm{x} \pm \mathrm{s}$, and intergroup comparisons were performed using an independent sample $t$ test. Qualitative data were expressed as percentages, and intergroup comparisons were performed using $\chi^{2}$ test.
$\mathrm{P}<0.05$ indicated statistically significant difference.

\section{Results}

The results of comparison of the ONS compliance rate between the two groups of patients after one month of care interventions ( $\mathrm{n} \%)$ are shown in Table 1.

Table 1. Comparison of ONS compliance rate between both patient groups, $(n \%)$.

\begin{tabular}{lllll}
\hline Degree of compliance & Control group $(\mathbf{n}=\mathbf{3 4})$ & Observation group $(\mathbf{n}=\mathbf{3 4})$ & $\mathbf{X}^{\mathbf{2}}$ & $\mathbf{p}$ \\
\hline Difference & $14(41.18)$ & $6(17.65)$ & 7.028 & 0.029 \\
Normal & $8(23.53)$ & $8(23.53)$ & & \\
Good & $12(35.29)$ & $20(58.82)$ & & \\
\hline
\end{tabular}

+ Note: $\mathrm{P}<0.05$.

The results of comparison of quality of life scores between the two patient groups $(\mathrm{x} \pm \mathrm{s})$ before implementing care interventions are shown in Table 2 .

Table 2. Comparison of quality of life scores between the two patient groups before care interventions $(x \pm s)$.

\begin{tabular}{|c|c|c|c|c|}
\hline Dimension & Control group & Observation group & $\mathbf{t}$ & $\mathbf{P}$ \\
\hline Physical functioning & $22.32 \pm 3.19$ & $22.18 \pm 3.97$ & 0.139 & 0.867 \\
\hline Psychological functioning & $33.87 \pm 2.51$ & $33.09 \pm 2.34$ & 0.597 & 0.603 \\
\hline Social functioning & $19.59 \pm 2.21$ & $19.78 \pm 2.08$ & 0.091 & 0.896 \\
\hline Common symptoms and side effects & $25.76 \pm 3.88$ & $26.36 \pm 4.87$ & 0.406 & 0.543 \\
\hline Specific domain & $45.34 \pm 3.61$ & $45.99 \pm 3.06$ & 0.971 & 0.392 \\
\hline Overall quality of life & $146.77 \pm 7.09$ & $147.41 \pm 6.77$ & 0.337 & 0.606 \\
\hline
\end{tabular}

+ Note: $\mathrm{P}<0.05$

The results of comparison of quality of life scores between the two patient groups $(\mathrm{x} \pm \mathrm{s})$ after one month of care interventions are shown in Table 3.

Table 3. Comparison of quality of life scores between the two patient groups after one month of care interventions ( $x \pm s$ )

\begin{tabular}{|c|c|c|c|c|}
\hline Degree of compliance & Control group $(n=34)$ & Observation group $(n=34)$ & $\mathbf{X}^{2}$ & $\mathbf{p}$ \\
\hline Difference & $14(41.18)$ & $6(17.65)$ & 7.028 & 0.029 \\
\hline Normal & $8(23.53)$ & $8(23.53)$ & & \\
\hline Good & $12(35.29)$ & $20(58.82)$ & & \\
\hline Dimension & Control group & Observation group & & $\mathbf{P}$ \\
\hline Physical functioning & $19.12 \pm 2.08^{\Delta}$ & $21.82 \pm 2.34$ & & $<0.002$ \\
\hline Psychological functioning & $29.42 \pm 2.23^{\Delta}$ & $36.96 \pm 3.11 *$ & & $<0.010$ \\
\hline Common symptoms and side effects & $20.47 \pm 2.81^{\Delta}$ & $25.12 \pm 3.79$ & & $<0.001$ \\
\hline Specific domain & $40.55 \pm 2.93^{\Delta}$ & $47.76 \pm 3.63 *$ & & $<0.030$ \\
\hline Overall quality of life & $127.44 \pm 6.23^{\Delta}$ & $152.53 \pm 6.42 *$ & & $<0.001$ \\
\hline
\end{tabular}

+ Note: $\mathrm{P}<0.05$

\section{Discussion}

Elderly patients with cirrhosis exhibit a worse physical constitution and medication compliance compared with their younger counterparts. In fact, this is a common phenomenon among the elderly. However, complications in late stage cirrhosis are extremely dangerous for patients and often endanger their health and life. Ultimately, this is closely associated with patients' malnutrition status. Studies across the globe have shown [10-12]. that ONS has a beneficial effect on alleviating malnutrition in elderly patients with cirrhosis. Although it has been reported that [13]. ONS was not effective among certain elderly patients and might even reduce patients' quality of life, it has also been shown that [14]. this was due to elderly patients' compliance. In China, many researchers have learned from the extensive experience of their international peers, while also taking into consideration the national clinical conditions. They have adopted the shared decision-making intervention to improve poor ONS compliance among elderly patients with cirrhosis in an attempt to improve patients' quality of life. The study by Li Yu et al [15]. showed that family members were important participants in the decision-making process for patient surgeries, but the degree of involvement by family members exceeded what was 
desired by patients. The study by Chen Peijuan et al [16]. also demonstrated that compared with standard nutrition treatment, the implementation of appropriate care interventions with ONS improved patients' compliance and energy intake as well as their quality of life. Therefore, to improve ONS compliance and quality of life among elderly patients with cirrhosis, selecting appropriate care interventions is crucial.

The care intervention via shared decision-making utilized in the present study is the latest clinical care model adopted globally [17-18]. This care model refers to the joint participation of healthcare workers, patients, and family members in formulating the most appropriate care plan. Shared decision-making has been broadly applied in practice and has always achieved satisfactory treatment effectiveness. In this study, shared decision-making was used among elderly patients with cirrhosis who received ONS to improve compliance and quality of life. The results in Table 1 showed that one month after implementing the care interventions in the both patient groups, the number of patients in the observation group who showed compliance was significantly higher than that in the control group. This indicates that the implementation of shared decision-making is extremely beneficial in improving ONS compliance in elderly patients with cirrhosis. In addition, patients and their families in the observation group were also more willing to follow each treatment in the plan and tended to be more satisfied. The results in Table 2 showed that the differences in quality of life scores between the control and observation groups were not statistically significant $(\mathrm{P}>0.05)$ before care interventions, and were not comparable.

The results in Table 3 showed that after one month of care interventions, the scores for overall quality of life and other dimensions were significantly higher in the observation group than in the control group $(\mathrm{p}<0.05)$. Comparisons within the control group showed that the scores for overall quality of life and other dimensions were significantly lower after the interventions compared with before the interventions $(p<0.05)$. There may be several reasons for these findings among control group patients who received routine care interventions. First, patients and their families were tired from previous care measures; and second, treatments based on previous standardized care plans can result in a sense of oppression that will more likely lead to reverse psychology in patients and their families. With the exception of the physical functioning and common symptoms and side effect domains, patients in the observation group exhibited significantly higher scores in other dimensions after the interventions compared to before the interventions $(p<0.05)$. The results of the present study demonstrate that care interventions via shared decision-making have an extremely positive effect on improving ONS compliance in elderly patients with cirrhosis. As ONS is the primary measure for treating malnutrition in elderly patients with cirrhosis, the implementation of shared decision-making has greatly improved the patients' quality of life. This care approach partially relieves patients and their families' uncertainties about the disease. It also eliminates the contradictions between physicians and patients, allowing patients and their families to place more trust in healthcare workers. Furthermore, this approach can enhance communication and interactions between physicians and patients. This prevents false disclosures from patients that can lead to misjudgments by physicians, which may prolong patients' disease courses and even threaten the patients' lives.

It is evident from the above clinical research and result analyses that patients are willing to accept this care model. By showing the patients and their families the relevant results, they also received positive feedbacks, which greatly strengthened the degree of voluntary cooperation by patients and their families. Barry et al [19]. have also demonstrated that shared decision-making can increase patient and family members' trust in healthcare workers. Although the present study has provided additional proof that shared decision-making is worthwhile for wide adoption in current clinical care work, this study has limitations. The sample size was small, and the specific implementation plan of shared decision-making was not ideal. It is necessary to conduct further investigations using a larger sample size. In addition, the cultural backgrounds of patients and their families in this study varied. The different degrees of understanding by patients and family members could also lead to various issues during the study, the impact of which needs to be further investigated.

\section{Conclusion}

This article through to 34 cases of aged patients with cirrhosis nutrition care Shared decision-making , analyses that patients are willing to accept this care model. By showing the patients and their families the relevant results, they also received positive feedbacks, which greatly strengthened the degree of voluntary cooperation by patients and their families, ONS can improve greatly the patients compliance, which could help to correct and strengthen nutrition in elderly patients with cirrhosis of the liver, can avoid or improve the damage factors for ONS to patients and poor clinical outcome, has important significance on the prognosis of patients with cirrhosis, thus improve the life quality of patients.

\section{Acknowledgements}

We would like to thank Editage (www.editage.cn) for English language editing.

\section{References}

[1] Guojun, L. Jiemin, H. Hehui, Z. et al. (2011). Clinical characteristics of hospitalized patients with cirrhosis and concurrent nosocomial infection [J]. Chinese Journal of Nosocomiology, 21 (4): 685-686.

[2] Cong, M. H. Li, S. L. Cheng, G. W. et al. (2015). An Interdisciplinary Nutrition Support Team Improves Clinical and Hospitalized Outcomes of Esophageal Cancer Patients with Concurrent Chemoradiotherapy. Chin Med J (Engl), 128 (22): 3003-3007. 
[3] Lihong, Z. Xiaorong, Z. (2019). Effects of high-quality care combined with intensified parenteral nutrition support on immune function and quality of life in patients with gastric cancer and intestinal obstruction [J]. Hebei Medical Journal, 41 (09): 1431-1433+1437.

[4] Torda, T. A. Pybus, D. A. (1984). Extradural administration of morphine and bupivacaine controlled comparison. $\mathrm{Br} \mathrm{J}$ Anaesth, 56: 141-146.

[5] Simon, D. Schorr, G. Wirtz, M. et al. (2006). Development and first validation of the shared decision-making questionnaire (SDM-Q) [J]. Patient Educ Couns, 63 (3): 319-327.

[6] Smith, M. A. (2016). The role of shared decision making in patient-centered care and orthopaedics, J. Orthop Nurs, 35 (3): 144-149.

[7] Zaiying, L. Nanshan, Z. (2008). Internal Medicine [M]. 7th Edition. Beijing: People's Medical Publishing House, 402-412.

[8] Chinese Society for Parenteral and Enteral Nutrition. (2017). Guidelines for nutritional support in cancer patients. Chinese Journal of Surgery, 55 (11): 801- 829

[9] Jiahong, L. Chonghua, W. Qiong, M. et al. (2007). Development and evaluation of the quality of life instrument for patients with head and neck cancer QLICP-HN in China. Modern Preventive Medicine, 34 (21): 4023-4025.

[10] Lee, J. L. Leong, L. P. Lim, S. L. (2016). Nutrition intervention approaches to reduce malnutrition in oncology patients: a systematic review. Support Care Cancer, 24 (1): 469-480.

[11] Ravasco, P. Monteiro-Grillo, I. Marques, V. P. et al. (2005). Impact of nutrition on outcome: a prospective randomized controlled trial in patients with head and neck cancer undergoing radiotherapy. Head Neck, 27 (8): 659-668.
[12] Qunying, Z. Zhongyu, J. (2018). Effect of adequate nutritional support on nutritional status and quality of life of cancer patients after chemotherapy. Parenteral and Enteral Nutrition, 25 (3): $138-141$.

[13] Arends, J. Bachmann, P. Baracos, V. et al. (2017). ESPEN guidelines on nutrition in cancer patients. Clin Nutr, 36 (1): 11-48.

[14] Talwalkar, J. A. (2011). Evening administration of long-acting beat-blockers for primary prophylaxis for primary primary prophylaxis in cirrhosis: an effective strategy. Am J Gastroenterol, 106: 545.

[15] Yu, L. Zhixia, Y. Peiyu, L. Li, L. (2016). Current status and factors influencing participation in decision-making for surgery in informed patients with primary liver cancer [J]. Journal of Nursing (China), 23 (18): 6-11.

[16] Peijuan, C. Li, W. Wenzhi, C. (2017). Effects of integrated medical care on the compliance to jaw-opening exercise and quality of life in patients with nasopharyngeal carcinoma. Guangdong Medical Journal, 38 (12): 1941-1943+1947.

[17] Elwyn, G. Frosch, D. L. Kobrin, S. (2016). Implementing shared decision-making: consider all the consequences. Implement Sci, 11: 114.

[18] Shay, L. A. Lafata, J. E. (2015). Where is the evidence? A systematic review of shared decision making and patient outcomes. Med Decis Making, 35 (1): 114-131.

[19] Barry, M. J. Edgman, L. S. (2012). Shared Decision Making-pinnacle of Patient-centered Care. J N Eng J Med, 366 (9): 780-781. 